\title{
Verification data for electron beam dose algorithms
}

\author{
Almon S. Shiu, Samuel Tung, and Kenneth R. Hogstrom \\ Department of Radiation Physics, The University of Texas M. D. Anderson Cancer Center, \\ 1515 Holcombe Blvd., Houston Texas 77030 \\ John W. Wong, Russell L. Gerber, William B. Harms, and James A. Purdy \\ Division of Radiation Oncology, Mallinckrodt Institute of Radiology, Washington University School of \\ Medicine, 510 South Kingshighway, St. Louis, Missouri 63110 \\ Randall K. Ten Haken, Daniel L. McShan, and Benedick A. Fraass \\ Department of Radiation Oncology, University of Michigan Medical Center, 1500 E. Medical Center Dr., \\ Ann Arbor, Michigan 48109
}

(Received 20 June 1991; accepted for publication 10 December 1991)

The Collaborative Working Group (CWG) of the National Cancer Institute (NCI) electron beam treatment planning contract has performed a set of 14 experiments that measured dose distributions for 28 unique beam-phantom configurations that simulated various patient anatomic structures and beam geometries. Multiple dose distributions were measured with film or diode detectors for each configuration, resulting in 78, 2-D planar dose distributions and one, 1-D depth-dose distribution. Measurements were made for 9- and 20-MeV electron beams, using primarily $6 \times 6$ - and $15 \times 15-\mathrm{cm}$ applicators at several SSDs. Dose distributions were measured for shaped fields, irregular surfaces, and inhomogeneities (1-D, 2-D, and 3-D), which were designed to simulate many clinical electron treatments. The data were corrected for asymmetries, and normalized in an absolute manner. This set of measured data can be used for verification of electron beam dose algorithms and is available to others for that purpose.

Key words: electron beam dosimetry, irregular fields, inhomogeneities, measured dose distributions, algorithm verification, treatment planning

\section{INTRODUCTION}

In algorithm verification, calculated dose distributions are compared with measured dose distributions for an entire 3-D volume or for selected portions, e.g., planar, linear, or point dose distributions. The purpose of algorithm verification is twofold: (1) to quantify the accuracy of the dose algorithm as a function of position in the beam for specific beam geometries, patient geometries, and model parametrizations; and (2) to validate the algorithm. Both are necessary prior to using a dose calculation algorithm for patient treatment planning.

In a recent Collaborative Working Group contract on high-energy electron beam treatment planning (ECWG), verification of the validity and accuracy of 3-D electron beam dose calculation algorithms was a crucial component of the work of the group. [High Energy 3-D Electron Beam Treatment Planning Cooperative Working Group, consisting of the Mallinckrodt Institute of Radiology at Washington University in St. Louis (WU), the University of Michigan Medical Center in Ann Arbor (UM), and the University of Texas M. D. Anderson Cancer Center in Houston (UT), supported by National Cancer Institute Contracts N01-CM-67913, 4, and 5.] In order to enable the algorithm verifications, an extensive data set of electron beam dose distributions was measured and analyzed. The full data set was then used by members of the ECWG to validate their individual algorithms. We anticipate subsequent papers, which address the quantitative results of the algorithm verification exercise, to be published by the three institutions separately. The ECWG also performed clinical treatment planning exercises involving electron beam treatment of several sites commonly treated with electron beams: the intact breast, post-mastectomy chest wall, larynx, craniospinal irradiation, orbit, parotid, and nasal vestibule. Therefore, in contrast to some earlier work on algorithm verification, which concentrated on experimental geometries designed specifically for testing the physics of the algorithms, ${ }^{1,2}$ the ECWG designed verification tests to simulate situations that would be encountered in the clinical treatment planning part of the ECWG studies.

Dose distributions from a total of 28 different experimental configurations were measured in this work. Each of the experiments was designed to test either (1) the fundamental characteristics of the electron beam calculation algorithm, or (2) the behavior of the algorithm in a situation applicable to a particular clinical site. Dose distributions were measured for situations including variation of energy, electron applicator, SSD, field shaping, irregular surfaces, and inhomogeneities (1-D, 2-D, and 3-D) using air, hung, and bone tissue substitutes. ${ }^{3,4}$ Detailed analysis of the measured data has generated a self-consistent data set that can be used for electron beam dose calculation verification studies. The data set is available on magnetic tape to interested institutions. (Address ECWG data set tape requests to A. S. Shiu, Ph.D., Department of Radiation Physics, Box 94, The University of Texas M. D. Anderson Cancer Ctr., 1515 Holcombe Blvd., Houston, TX 77030.) This paper describes the irradiation conditions and phantom ge- 
TABLE I. Electron properties of various tissue substitutes.

\begin{tabular}{|c|c|c|c|c|c|c|}
\hline Tissue substitute & Styrofoam $^{b}$ & $\begin{array}{c}\text { Lung } \\
\text { Substitutes }^{\mathrm{c}}\end{array}$ & Water & $\begin{array}{l}\text { Electron } \\
\text { solid } \\
\text { water }^{c, d}\end{array}$ & $\begin{array}{c}\text { High-impact } \\
\text { polystyrene } \\
\quad \text { (white) } \\
\left(\mathrm{C}_{8} \mathrm{H}_{8_{n}}\right)+\mathrm{TiO}_{2}{ }^{\mathrm{f}}\end{array}$ & $\begin{array}{l}\text { SR4 hard } \\
\text { bone } \\
\text { substitute }^{\mathrm{e}}\end{array}$ \\
\hline $\begin{array}{l}\text { Physical density } \\
\qquad\left(10^{3} \mathrm{~kg} \mathrm{~m}^{-3}\right)\end{array}$ & 0.0265 & 0.294 & 1.000 & 1.022 & 1.055 & $1.692^{\mathrm{g}}, 1.699^{\mathrm{h}}$ \\
\hline $\begin{array}{l}\text { Linear collision } \\
\text { stopping power ratio }\end{array}$ & 0.0256 & 0.311 & 1.000 & 1.004 & 1.019 & $1.585^{\mathrm{g}}, 1.592^{\mathrm{h}}$ \\
\hline $\begin{array}{l}\text { Linear scattering } \\
\text { power ratio }\end{array}$ & 0.0210 & 0.292 & 1.000 & 0.901 & 0.829 & $2.103^{g}, 2.112^{\mathrm{h}}$ \\
\hline
\end{tabular}

${ }^{a}$ Ratio means value of tissue substitute relative to that of water.

${ }^{b}$ Compositions: $73.5 \%-100 \%$ polystyrene, $0-10 \%$ chlorodifluoro ethane, $0-10 \%$ polyethylene, $0-4.5 \%$ ethylchloride, and $0-2 \%$ hexabromocyclododecane (assumed $100 \%$ polystyrene for calculation of scattering and stopping powers).

${ }^{c}$ Available from Radiation Measurements, Inc., Middleton, Wisconsin.

${ }^{\mathrm{d}}$ Reference 11.

${ }^{\mathrm{e}}$ Reference 12.

${ }_{3} \% \mathrm{TiO}_{2}$, may vary with manufacture.

${ }^{8}$ For 2-D inhomogeneity.

${ }^{\mathrm{h}}$ For L-shaped bone.

ometries for the experiments, the dosimetry measurement techniques, the processing of the data, and aspects of the resulting data set.

\section{MATERIALS AND METHODS}

\section{A. General experimental design}

The Varian Clinac 1800 was selected from the list of machines available from each of the three institutions for making the data set, as that machine was common to two of the participating institutions. A detailed comparison of the features of all the machines available to the ECWG for this study showed the two machines to have nearly identical dosimetric characteristics. In addition, the members of the ECWG believed that the Clinac 1800 data would be the most challenging to fit using the pencil beam electron calculation algorithms which were based on the theory of Hogstrom et al. ${ }^{5,6}$ This is due in part to the design by Varian of the electron applicator and beam flattening system. At the highest energy, $20 \mathrm{MeV}$, the bremsstrahlung dose component is small compared to that from other machines using double scattering foil systems, which is speculated to occur because of a thinner primary scattering foil thickness. However, these results sacrifice flatness for the broad, high-energy beams that are utilized for craniospinal irradiation. The use of minimal scattering foil thickness requires some additional beam flattening being provided by scatter from the applicator walls. There is also significant leakage of primary electrons through the applicator walls, ${ }^{7,8}$ which tests dose calculation well outside the field. Both applicator leakage and scatter are not easily modeled and can result in a stringent test of any electron algorithm.

Two energies, 9 and $20 \mathrm{MeV}$, were selected, as it was expected that results from a high and low energy would be sufficient to illustrate any energy dependent disagreement of the algorithms to data. Also, $20 \mathrm{MeV}$ is in the energy range often used for craniospinal irradiation,, 10 and 9 $\mathrm{MeV}$ is an energy often used for chest wall or eye irradia- tion. Applicator sizes of $15 \times 15 \mathrm{~cm}$ and $6 \times 6 \mathrm{~cm}$ were used for the majority of the measurements.

The coordinate system used for data measurements was defined as follows: the $+Z$ direction is the same as the direction of the beam; the $+Y$ direction is away from the gantry; and the $+X$ direction is such as to make the coordinate system left handed. The origin of the coordinate system is on the beam central axis at isocenter. In specifying planar dose distributions, the axial plane parallel to the $X$ axis is defined as the transverse plane and that parallel to the $Y$ axis is defined as the radial plane.

A general approach to data collection was used. In general, most experiments involved data acquisition in a number of planes: (1) axial plane through the central axis of the beam, (2) a second axial plane $2 \mathrm{~cm}$ inside the field edge, (3) two "beam's eye view" planes (a BEV plane is orthogonal to the beam central axis), at depths of $2 \mathrm{~mm}$ and the depth of the $90 \%$ of absorbed dose maximum $\left(R_{90}\right)$ along the central axis of the open field. As described below, the axial measurements were made with diode detectors in a water phantom, while the BEV planes were measured with film.

\section{B. Measurement methods and phantom materials}

All measured data used for the algorithm verification were obtained in one of two ways: (1) diode detectors in a water phantom, or (2) XTL-2 film in a solid water phantom, with the exception of one depth-dose curve in lung substitute measured using thermoluminescent dosimeters (TLD). The physical densities, linear collision stopping power ratios, and linear scattering power ratios ${ }^{11,12}$ (with respect to water) of all the water-equivalent material (electron solid water ${ }^{11}$ and high impact polystyrene ${ }^{12}$ ) and inhomogeneities [styrofoam (Dow Chemical Co., Midland, MI 48674), lung, and bone substitutes] are listed in Table I. Only selected examples of irradiation conditions are described in Sec. III. 


\section{Water phantom data}

All water phantom data were collected using a diode detector (Therados unshielded p-type silicon diode) in an RFA-3 water phantom system. Work published previously ${ }^{13}$ has shown that the diode detector dosimetry agrees well with parallel plate (NACP) and cylindrical (Farmer type, RK, PTW) ion chambers, TLD, and film dosimetry. The diode was located $0.3 \mathrm{~mm}$ below the surface of the detector, and that point was taken as the effective point of measurement. The active area of the detector is $2 \times 2 \mathrm{~mm}$.

The RFA-3 water phantom system was interfaced to a microVAX II computer for data acquisition. The computer controlled the 3-D position of the field probe with respect to the origin and determined the relative dose for the field detector and a reference detector by averaging several readings. After individual scans, the measured data and other identifying information were written automatically to an ASCII file.

For each depth-dose or profile scan, the dose at the predefined normalization point was read automatically before and after each scan. The system was configured to move the detector in its smallest digital step size $(\sim 0.3$ $\mathrm{mm}$ ) for each individual dose reading. For a single dose reading, the system averaged automatically the dose values obtained from several $(>5)$ readings of the scanning detector relative to the reference detector, until the variance in the readings was less than a value specified previously (typical default value was $0.5 \%$ ). Relative to each data point, the next data point was obtained when (a) the dose reading changed by more than $1 \%$ from the previous data point or (b) the new position was more than $5 \mathrm{~mm}$ (for the depth dose) or $10 \mathrm{~mm}$ (for profiles) from the location of the previous "datapoint." This method allowed the system to take more closely spaced datapoints in the areas of steep dose gradient, while decreasing the number of datapoints in the areas of low-dose gradient.

The two-dimensional dose distribution was then generated by using the depth-dose curve to interpolate between the various profiles (typical $6 \sim 8$ profiles measured at various depths) similar to the method of Milan and Bentley. ${ }^{14}$ These data were then used to generate a two-dimensional dose distribution on a rectilinear grid (typical grid spacing $2.5 \times 2.5 \mathrm{~mm}$ ). The two-dimensional dose distributions are the data to be distributed to the interested institutions.

\section{Film/solid water data}

XTL-2 film was used for the measurements made in BEV planes and other selected geometries. The film was sandwiched in an electron solid-water film cassette, ${ }^{15}$ which was sandwiched between slabs of solid water. Each film was irradiated with approximately $6 \mathrm{cGy}$, and then developed. Each film was digitized using a Scanditronix RFA-7 film densitometer system, by scanning with an aperture of $2-\mathrm{mm}$ diameter on a $2 \times 2-\mathrm{mm}$ grid of points. Appropriate $H$ and $D$ curve conversions from net optical density to dose were applied to each scan.

\section{TLD data}

Thermoluminescent dosimetry was used to measure the depth-dose curve in a lung substitute phantom. Each TLD consisted of approximately $27 \mathrm{mg}$ of Lithium Fluoride TLD-100 powder enclosed in a sealed cellophane pack. The active area of the dosimeter was approximately $4 \times 4$ $\mathrm{mm}^{2}$ and $0.5-\mathrm{mm}$ thick. To calibrate the thermoluminescence (TL) sensitivity, a set of reference TLDs, placed at a depth of maximum dose in a solid water phantom, was irradiated to a dose of $300 \mathrm{cGy}$ during the measurement session for a $15 \times 15-\mathrm{cm}$ field at $100-\mathrm{cm}$ SSD. Details of the conversion from TL to dose have been described before. ${ }^{16}$ Three readings per depth measurement indicated a maximum variation of $2 \%$ about the mean.

\section{Data reduction}

Before a data set could be used for the algorithm verification and comparison, it was essential that all the measured data be consistent for each given irradiation condition. To assure that the data set met this requirement, significant effort was made to process the data. The data processing can be divided into several categories: data normalization, data manipulation, and data transfer.

During analysis, all the measured dose distributions were renormalized so that the maximum central-axis dose for the reference geometry was equal to $100 \%$. The reference geometry for each experiment consisted of the open applicator (no field shaping) irradiating a water phantom at $100-\mathrm{cm}$ SSD. This normalization method was chosen because it is frequently used in treatment planning systems. As the dose per monitor unit was known for the reference geometry, this method allowed easy calculation of monitor units required to deliver a specified dose for the irradiation geometry. By normalizing in this manner, it is possible to compare absolute rather than relative dose distributions. During the design of each experiment, a normalization point was defined for each measurement (depth profile, off-axis profile, or BEV planar dose distribution) so that the various measurements could all be normalized to create a self-consistent dose distribution. Whenever possible, common points (e.g., line of interaction) between planes of data were used to assure the consistency of the data.

In the process of generating the self-consistent dose distributions, a number of different kinds of data manipulation were required. These include (1) translation to account for detector and/or coordinate system misalignment relative to the central axis or to the phantom surface, (2) rotations to align the detector orientation (typically the dose distribution scanned from film) to the desired measurement coordinate system, and (3) repair of obviously bad data. It should be emphasized that the data manipulation was a process to correct measurement errors or artifacts, which could be due to alignment errors during setup or scanning of the film.

\section{EXPERIMENTS}

Fourteen different experiments were performed, and each experiment had from one to four unique irradiation 
TABLE II. Algorithm verification experiment configuration summary.

\begin{tabular}{|c|c|c|c|c|c|c|c|c|}
\hline \multirow[b]{2}{*}{ Exp } & \multirow[b]{2}{*}{ Phantom } & \multirow{2}{*}{$\begin{array}{l}\text { SSD } \\
(\mathrm{cm})\end{array}$} & \multirow{2}{*}{$\begin{array}{l}\text { Energy } \\
(\mathrm{MeV})\end{array}$} & \multirow{2}{*}{$\begin{array}{l}\text { Applicator } \\
(\mathrm{cm} \times \mathrm{cm})\end{array}$} & \multirow{2}{*}{$\begin{array}{c}\text { Insert/block } \\
(\mathrm{cm} \times \mathrm{cm})\end{array}$} & \multicolumn{3}{|c|}{ Location of planes measured } \\
\hline & & & & & & $X(\mathrm{~cm})$ & $Y(\mathrm{~cm})$ & $Z(\mathrm{~cm})$ \\
\hline \multirow[t]{4}{*}{1} & water & 100 & 9 & $15 \times 15$ & - & - & $0.0,5.5$ & $0.2,2.8$ \\
\hline & & & & $6 \times 6$ & - & - & 0.0 & $0.2,2.6$ \\
\hline & & & 20 & $15 \times 15$ & - & - & $0.0,5.5$ & $0.2,6.1$ \\
\hline & & & & $6 \times 6$ & - & - & 0.0 & $0.2,4.7$ \\
\hline \multirow[t]{4}{*}{2} & water & 110 & 9 & $15 \times 15$ & - & - & 0.0 & $0.2,2.8$ \\
\hline & & & & $6 \times 6$ & - & - & 0.0 & $0.2,2.7$ \\
\hline & & & 20 & $15 \times 15$ & - & - & 0.0 & $0.2,6.1$ \\
\hline & & & & $6 \times 6$ & - & - & 0.0 & $0.2,5.5$ \\
\hline \multirow[t]{2}{*}{3} & water & 100 & 9 & $15 \times 15$ & $3 \times 12$ & 0.0 & 0.0 & $0.2,2.8$ \\
\hline & & & 20 & $15 \times 15$ & $3 \times 12$ & 0.0 & 0.0 & $0.2,6.1$ \\
\hline \multirow[t]{2}{*}{4} & water & 100 & 9 & $15 \times 15$ & house & - & $3.0,-3.0$ & $2.8,3.6$ \\
\hline & & & 20 & $15 \times 15$ & house & - & $3.0,-3.0$ & $6.1,8.2$ \\
\hline 5 & water & 110 & 20 & $25 \times 25$ & $5 \times 30$ diag. & $0.0,14.0$ & 0.0 & - \\
\hline 6 & solid water & 97 & 9 & $6 \times 6$ & eye & - & 0.0 & 2.6 \\
\hline \multirow[t]{2}{*}{7} & water $/ 30^{\circ}(2-\mathrm{D})$ & 104.3 & 9 & $15 \times 15$ & - & - & 0.0 & 0.2 \\
\hline & & & 20 & $15 \times 15$ & - & - & 0.0 & 0.2 \\
\hline \multirow[t]{2}{*}{8} & solid water/step (2-D) & $100 / 98$ & 9 & $15 \times 15$ & - & 1.0 & 0.0 & - \\
\hline & & & 20 & $15 \times 15$ & - & 1.0 & 0.0 & - \\
\hline \multirow[t]{2}{*}{9} & solid water/nose (3-D) & 9 & $15 \times 15$ & - & - & - & $0.0,-1.0,-3.0$ & $0.2,2.8$ \\
\hline & & & 20 & $15 \times 15$ & - & - & $0.0,-1.0,-3.0$ & $3.1,6.1$ \\
\hline 10 & solid water/lung( $1-D)$ & 100 & 9 & $15 \times 15$ & - & $\mathrm{CAX} \% \mathrm{DD}$ & - & 6.0 \\
\hline 11 & solid water/lung(2-D) & 100 & 20 & $15 \times 15$ & - & - & 0.0 & $5.0,7.0$ \\
\hline \multirow[t]{2}{*}{12} & water/bone (2-D) & 100 & 9 & $15 \times 15$ & - & - & 0.0 & - \\
\hline & & & 20 & $15 \times 15$ & - & - & 0.0 & - \\
\hline \multirow[t]{2}{*}{13} & water/air (2-D) & 100 & 9 & $15 \times 15$ & - & - & 0.0 & - \\
\hline & & & 20 & $15 \times 15$ & - & - & 0.0 & - \\
\hline \multirow[t]{2}{*}{14} & water/bone (3-D) & 100 & 9 & $15 \times 15$ & - & - & $1.0,-1.0$ & 2.8 \\
\hline & & & 20 & $15 \times 15$ & - & - & 1.0 & 6.1 \\
\hline
\end{tabular}

conditions for a total of 28 verification tests. The various experiments, along with the locations of each planar dose distribution, are listed in Table II. In the sections below, the salient points of each experimental setup are discussed; including the motivation behind the experiment and the differences between the general experimental schema described above and the details of the individual experiment.

\section{A. Experiment 1: Water phantom, standard SSD}

The most fundamental test of any radiation dose calculation algorithm is its ability to predict (or at least reproduce) the measured dose distribution in a water phantom at the standard treatment distance. Thus Experiment 1 measured the dose distributions for the four basic situations, small $(6 \times 6)$ and large $(15 \times 15)$ fields at low $(9$ $\mathrm{MeV})$ and high $(20 \mathrm{MeV})$ energies, with a standard $100-$ $\mathrm{cm}$ SSD setup. The air gap between the end face of the electron applicator and the surface of the phantom was 5 $\mathrm{cm}$ for the standard BEV planes were used for each case. Figure 1 illustrates the measurement conditions for the 20 $\mathrm{MeV}, 15 \times 15-\mathrm{cm}$ applicator irradiation.

\section{B. Experiment 2: Water phantom, extended SSD}

The significance of an algorithm's ability to predict the dose distribution at extended treatment distances is similar to those areas of interest described in Experiment 1. Of particular interest is an algorithm's ability to predict (1) the increase in penumbra due to the increased air gap, (2) the change in depth dose, and (3) the change in the dose distribution, both within the therapeutic portion of the beam (within the $90 \%$ isodose surface) and outside the beam (outside penumbra) due to major differences in lowenergy electrons penetrating or being scattered off the applicator walls. Thus another series of situations (small and large fields, low and high energy) were measured, but with the SSD set to $110 \mathrm{~cm}$ rather than $100 \mathrm{~cm}$. The air gap was $15 \mathrm{~cm}$ instead of $5 \mathrm{~cm}$ for this setup. Planar dose distributions were measured in the same positions as in the first experiment with the exception of the off-axis planar isodoses, which were 0.3 and $0.75 \mathrm{~cm}$ farther from central axis for the $6 \times 6$ and $15 \times 15-\mathrm{cm}$ applicators, respectively, to maintain the same distance from the beam's edge at the water's surface as the first experiment $(2 \mathrm{~cm})$.

\section{Experiment 3: Water phantom, rectangular field}

Patients are usually treated with a lead or lead-alloy (Cerrobend) collimator inserted into the electron applicator to define an arbitrary beam shape. Because most algorithms can model rectangular fields, the dose distribution beneath a rectangular field insert was first studied: Both 9and $20-\mathrm{MeV}$ electron beams were used with a $3 \times 12-\mathrm{cm}$ insert in a $15 \times 15-\mathrm{cm}$ applicator with a standard $100-\mathrm{cm}$ SSD. For each irradiation condition, four planar dose distributions were measured: two containing the central axis and the major axes (one at $Y=0$, one at $X=0$ ) and two 


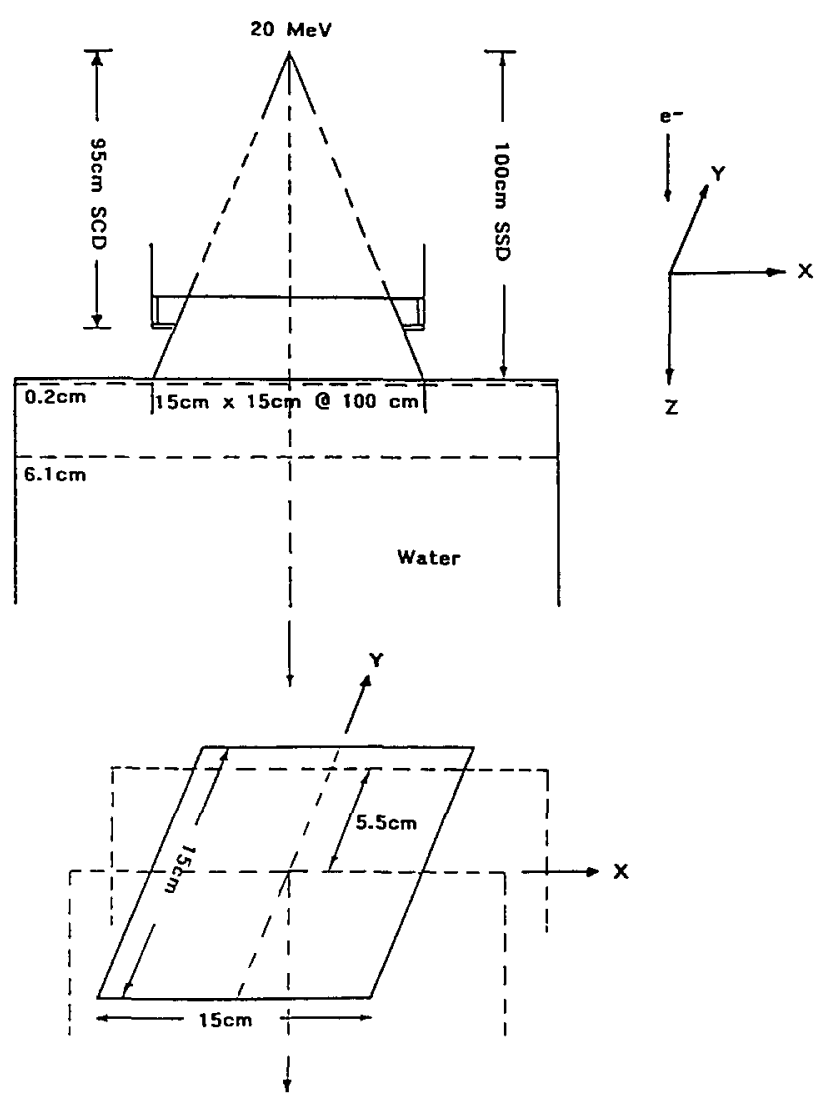

FIG. 1. Schematic of irradiation geometry of water phantom, $15 \times 15-\mathrm{cm}$ field, standard SSD for a $20-\mathrm{MeV}$ beam. The locations of the BEV dose distribution measurements for each depth are indicated by the dashed lines shown on the side view. The locations of the transverse dose distribution measurements for each plane are outlined by the dashed lines on the isometric view.

perpendicular to the central axis (BEV at $Z=0.2 \mathrm{~cm}$, $Z=R_{90}$ of the open applicator).

\section{Experiment 4: Water phantom, irregular field}

The majority of clinical electron fields are shaped irregularly using Cerrobend cutouts. The purpose of this test was to demonstrate an algorithm's ability to perform calculations for such fields. Figure 2 illustrates the geometry for the $20-\mathrm{MeV}$ irradiation condition. Referred to as the "house block," this shape was selected because its width was double-valued, because of the complexity created by the narrow appendage (chimney) which made a right angle near central axis, and by the triangular appendage (roof). These irregular features are severe and algorithm accuracy in the penumbra for this case can be compared to that of the regularly shaped collimation in the $-Y$ halfplane.

Both 9- and 20-MeV electron beams were used with the "house block" inserted into the $15 \times 15-\mathrm{cm}$ applicator; the water phantom was at the standard $100-\mathrm{cm}$ SSD. For each of the two irradiation conditions, four planar isodose distributions were measured-two parallel to central axis (at $Y=+3 \mathrm{~cm}, Y=-3 \mathrm{~cm}$ ) and two perpendicular to central axis ( $\mathrm{BEV}$ at $Z=R_{90}, Z=R_{50}$ for open applicator).
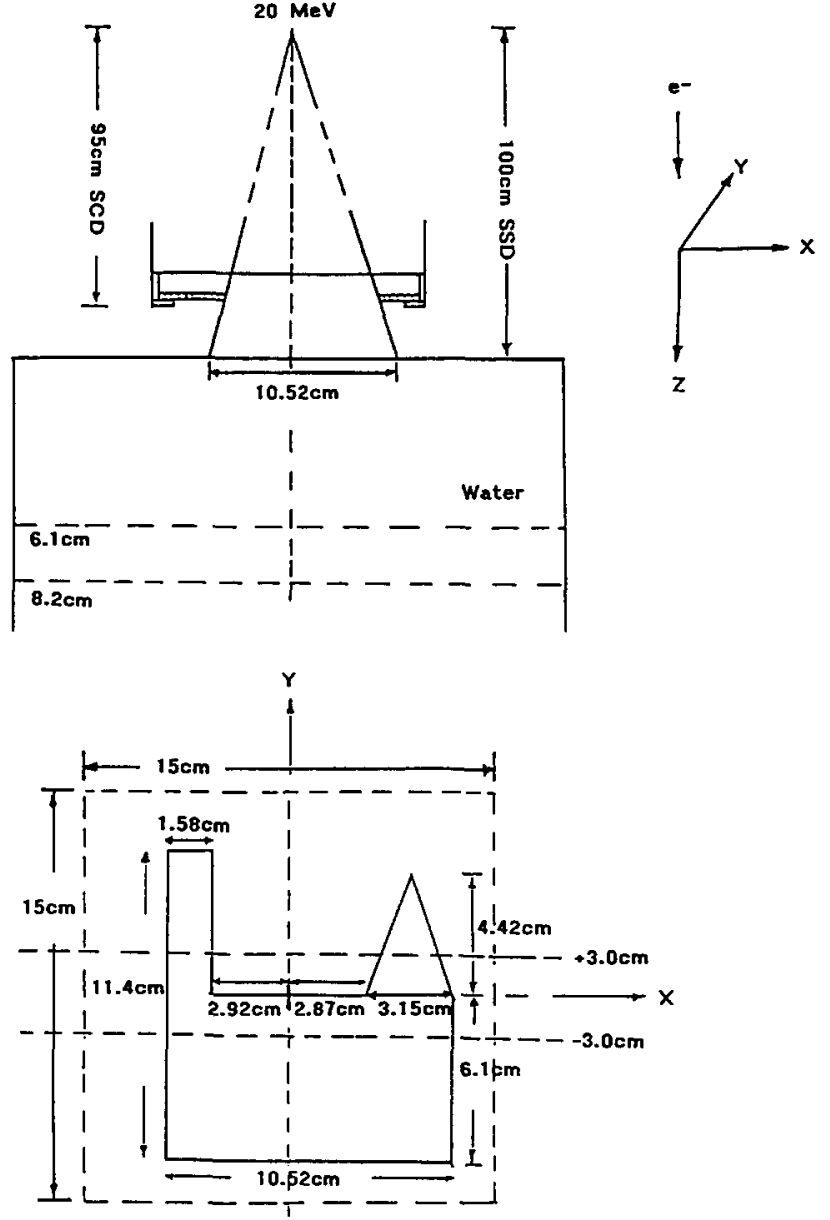

FIG. 2. Schematic of irradiation geometry of water phantom, irregular field, standard SSD for a $20-\mathrm{MeV}$ beam. The locations of the BEV dose distribution measurements for each depth are indicated by the dashed lines shown on the side view. The locations of the transverse dose distribution measurements for each plane are outlined by the dashed lines on the BEV. The irregular field shape is outlined by the solid line in the BEV.

\section{E. Experiment 5: Spinal field, craniospinal irradiation}

In craniospinal irradiation, the spinal cord can be irradiated with high-energy electrons (15-25 MeV) and a long electron field $(30-50 \mathrm{~cm}) .{ }^{9}$ If the linear accelerator produces a uniform beam over the diagonal of a $25 \times 25-\mathrm{cm}$ square, the spine of young children can usually be irradiated by a single field at an extended SSD, or in the case of adults, by no more than two abutted fields. In order to simulate these kinds of conditions, data were obtained for a $5 \times 30-\mathrm{cm}$ insert (defined at $100-\mathrm{cm}$ SSD) along the diagonal of the $25 \times 25-\mathrm{cm}$ applicator, with the collimator rotated so the long axis of the shaped field was along the principle axes of the water phantom system (see Fig. 3). The 20-MeV electron beam was chosen for this simulation, and the SSD was $110 \mathrm{~cm}$ for this setup (air gap $=15 \mathrm{~cm}$ ). Two planar dose distributions containing central axis were measured: (a) one along the length of the field $(Y=0)$ which is important for the abuttment dosimetry, and (b) one along the width of the field $(X=0)$ which is important for the target volume coverage. A third off-axis planar 


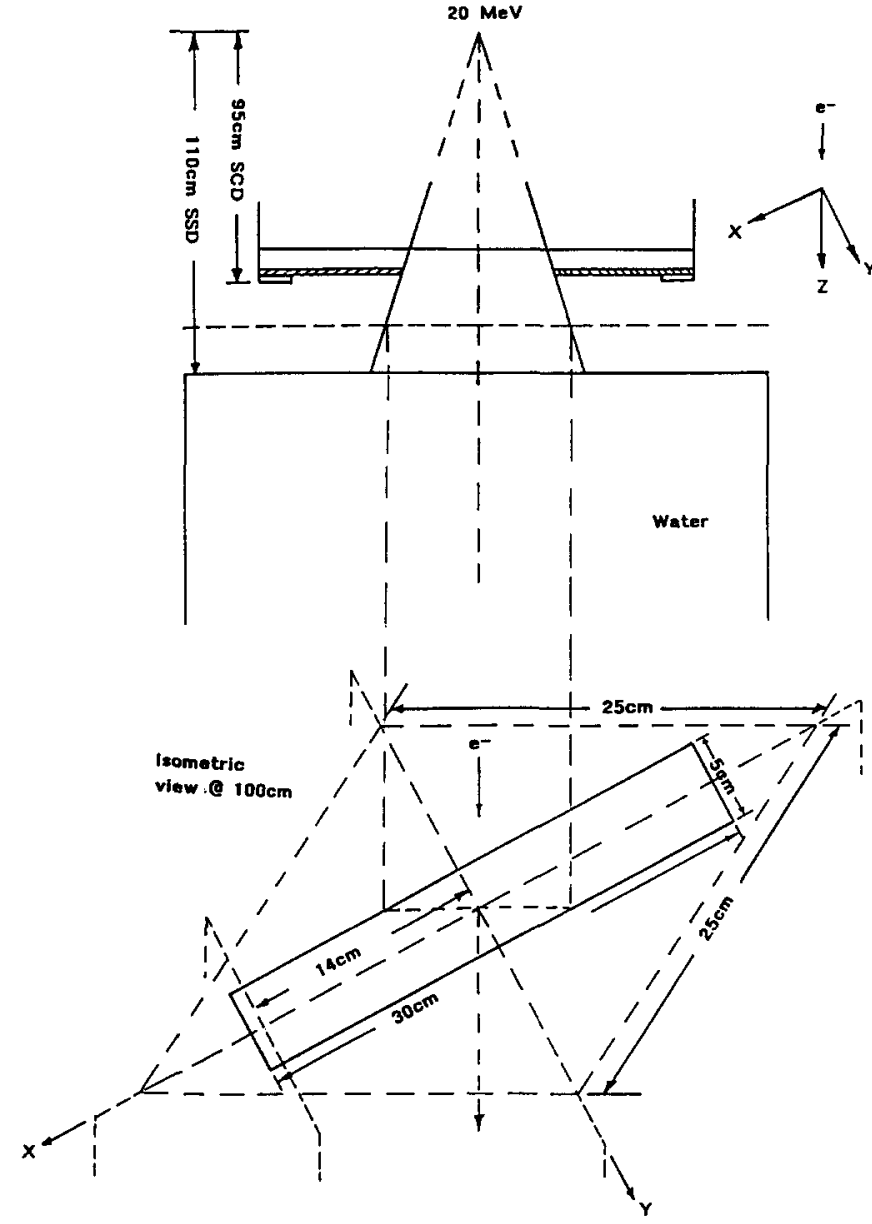

FIG. 3. Schematic of irradiation geometry of water phantom, rectangular spinal field, 110-cm SSD for a $20-\mathrm{MeV}$ beam. Measurement locations of the two planar dose distributions containing central axis and a third off-axis planar dose distribution are indicated by the dashed lines on the isometric view.

dose distribution ( $X=14 \mathrm{~cm}$, and parallel to central axis) was measured across the width of the field.

\section{F. Experiment 6: Eye-field irradiation}

In treatment of the eye, a nearly circular field is sometimes used with a central lens block. An anterior electron beam of about $10 \mathrm{MeV}$ can be used to irradiate the entire retina with a central-axis lens block $1 \mathrm{~cm}$ in diameter. Electrons scatter around the block, missing the lens but reaching the retina. ${ }^{17}$ The irradiation geometry for this test simulating the eye treatment procedure, is illustrated in Fig. 4. This test used a 9-MeV beam and a $5-\mathrm{cm}$ diameter insert (with a 1-cm diameter central-axis block) with the $6 \times 6-\mathrm{cm}$ applicator (field sizes specified at $100 \mathrm{~cm}$ from source). The water phantom was set at $97 \mathrm{~cm}$ SSD, as a reduced air gap of $2 \mathrm{~cm}$ minimizes lens dose for an actual eye irradiation. Because of radial symmetry, only one planar dose distribution in a plane containing the central axis was required $(Y=0)$. A single dose distribution in a plane perpendicular to the central axis ( $\mathrm{BEV}$ at $Z=2.6 \mathrm{~cm}, R_{90}$ of applicator) was also measured.

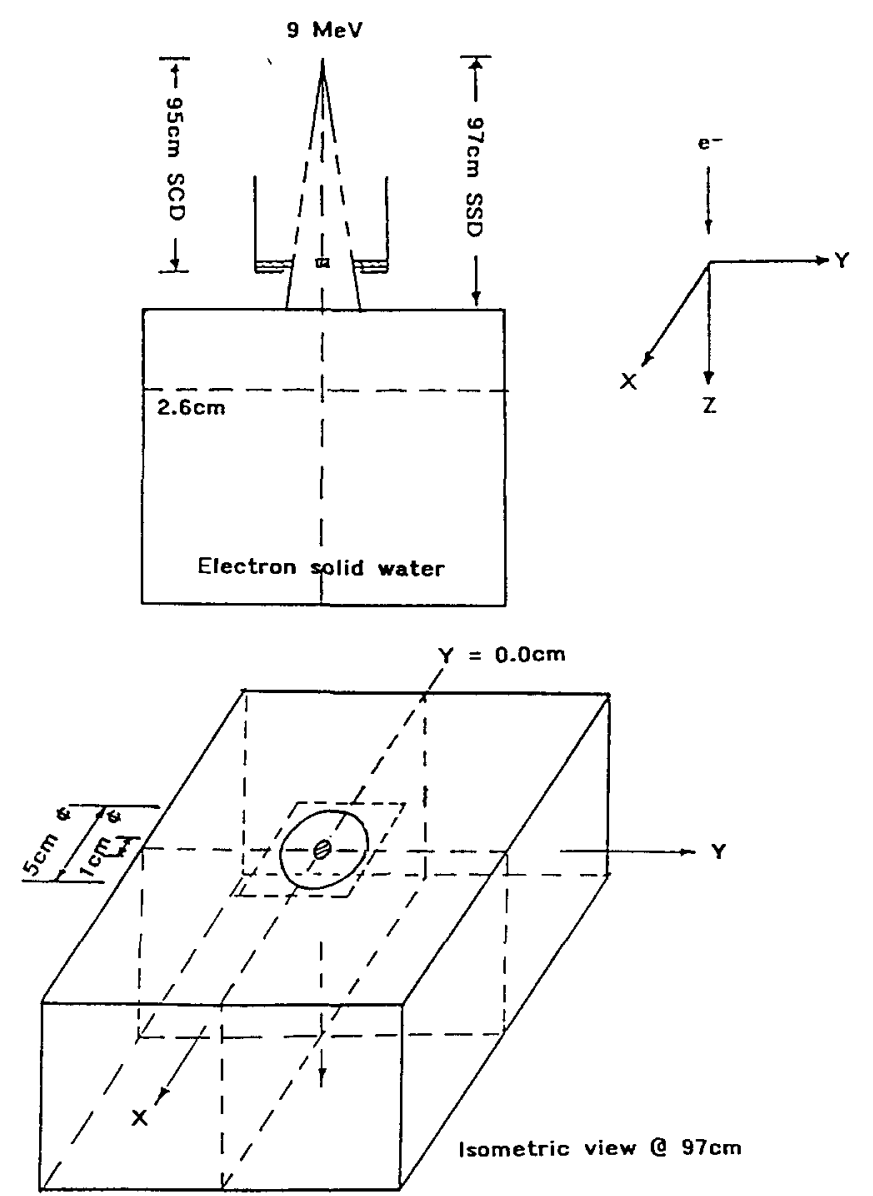

FIG. 4. Schematic of irradiation geometry of eye (retinoblastoma) measurements at $97-\mathrm{cm} \mathrm{SSD}$ for a $9-\mathrm{MeV}$ beam. The location of the BEV dose distribution measurement at a depth of $2.6 \mathrm{~cm}$ is indicated by the dashed lines is shown on the side view. The circular field with a central lens block and the location of the transverse planar dose measurements is illustrated on the isometric view.

\section{G. Experiment 7: Irregular surface (2-D), $30^{\circ}$ slope}

In irradiation of the chest wall, limbs, and neck, the patient's skin surface is usually not perpendicular to the incident beam. This angulation affects both penumbra ${ }^{6,18}$ and depth dose. ${ }^{19}$ To simulate the above irradiation geometries, this test used 9- and $20-\mathrm{MeV}$ electrons incident $30^{\circ}$ from the normal of the surface of a water phantom with the $15 \times 15-\mathrm{cm}$ applicator. A central-axis SSD of $104.3 \mathrm{~cm}$ was required to prevent the applicator from intersecting the water phantom. The $20-\mathrm{MeV}$ irradiation geometry is diagrammed in Fig. 5. Two planar dose distributions were measured, one in the plane of the gantry rotation $(Y=0)$ containing the central axis and one parallel to the surface of the phantom at a depth of $0.2 \mathrm{~cm}$.

\section{H. Experiment 8: Irregular surface (2-D), $90^{\circ}$ step}

In electron-beam radiotherapy, the patient's external surface often exhibits a steep step within the irradiation field. This may be due to normal anatomy (e.g., chin), a surgical defect, or an improperly partially bolussed field. Any step creates a hot/cold dose region; for a $90^{\circ}$ step and 


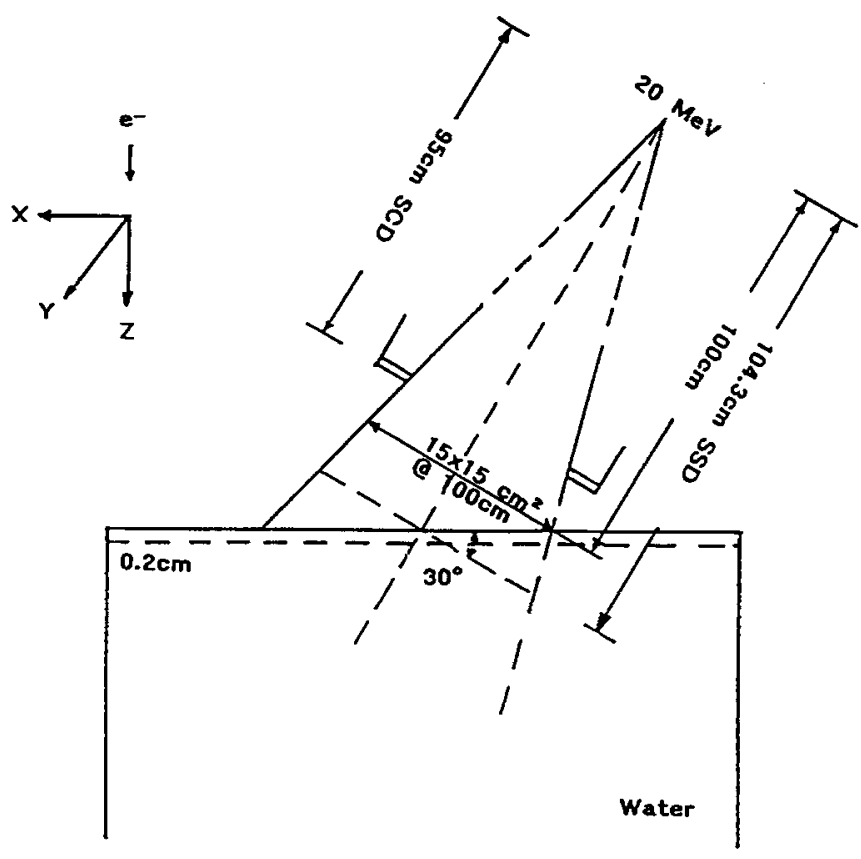

FIG. 5. Schematic of irradiation geometry of $30^{\circ}$ oblique incidence of a $20-\mathrm{MeV}$ beam into a water phantom at the central-axis SSD of $104.3 \mathrm{~cm}$. The location of the BEV dose distribution measurements at a depth of 0.2 $\mathrm{cm}$ is indicated by the dashed lines shown on the side view. The location of the dose distribution measurement in the principal transverse plane is indicated by the dashed lines on the isometric view.

parallel incident beam, this can theoretically result in a dose $\pm 50 \%$ of the intended dose. ${ }^{20}$

A 2-cm high $90^{\circ}$ stepped phantom was used for the measurement with the lower surface set at the 100-cm SSD for both energies; the $15 \times 15-\mathrm{cm}$ applicator was used for both irradiation conditions. The 9-MeV irradiation geometry is diagrammed in Fig. 6. Two planar isodose measurements were made: one contained the central axis and was perpendicular to the stepped surface $(Y=0)$; the other was parallel to the central axis and the vertical step and was located $1 \mathrm{~cm}$ from the step $(X=1 \mathrm{~cm})$.

\section{Experiment 9: Irregular surface (3-D), nose}

Tumors of the nose are often irradiated with anterior electron beams. ${ }^{18,21}$ If bolus is not utilized, then the irregular surface of the nose prevents lateral side-scatter equilibrium. A volume of increased dose (hot spot) will occur inferior and lateral to the nose, and a corresponding volume of decreased dose (cold spot) will occur beneath the nose. This can result in tumor underdose and overdose to normal tissue (e.g., upper gums).

To study the influence of the nose, a uniform semiinfinite water (or water equivalent) phantom with a right triangular cylinder $(2-\mathrm{cm}$ base, $3-\mathrm{cm}$ high, $4-\mathrm{cm}$ long) placed on the phantom's surface was irradiated with 20$\mathrm{MeV}$ electrons as illustrated in Fig. 7. Typically, nose tumors are irradiated with electrons in the range of 12-18 $\mathrm{MeV}$, so the extremes, both 9- and $20-\mathrm{MeV}$ electrons, were used for measurements. Dose distributions were measured in planes parallel to the central axis and perpendicular to
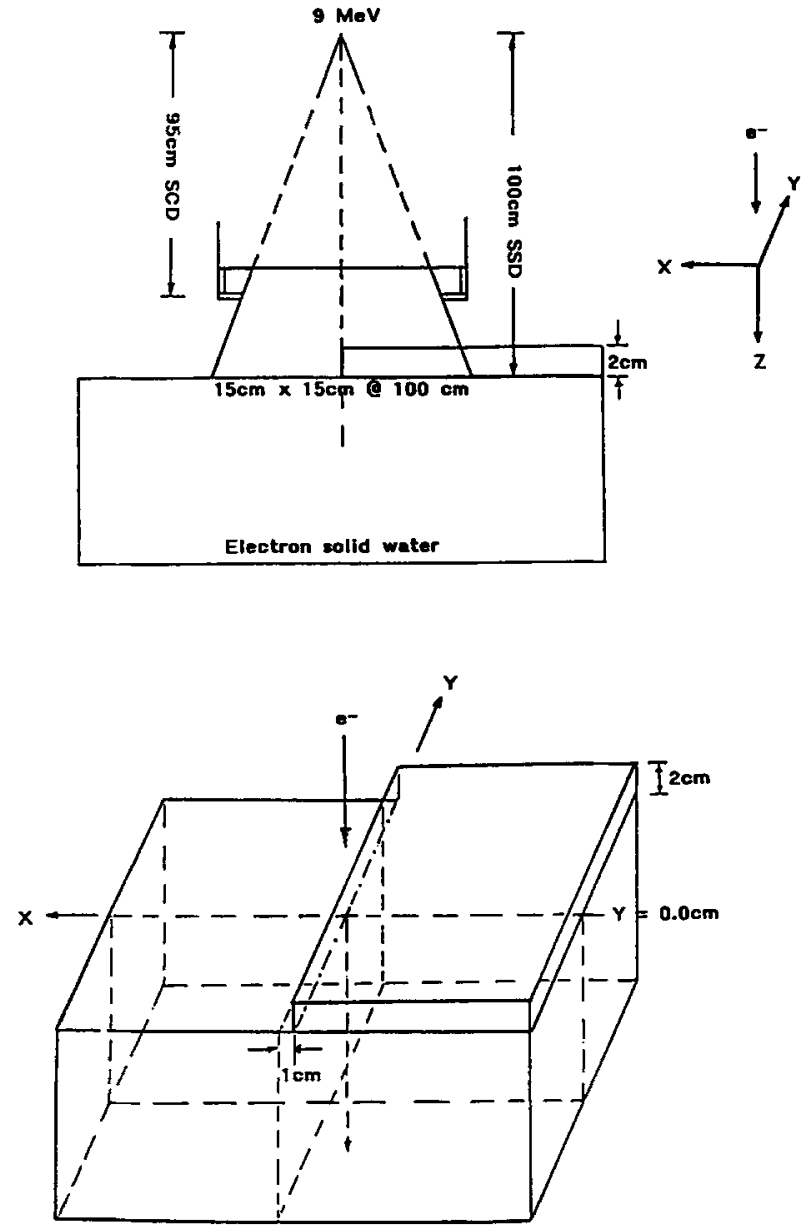

FIG. 6. Schematic of irradiation geometry of a $2-\mathrm{cm}, 90^{\circ}$-stepped phantom for a 9-MeV beam. The locations of the dose distribution measurements in the principal transverse plane and in the plane $1 \mathrm{~cm}$ away from the step and parallel to the principal radial plane are indicated by the dashed lines on the isometric view.

the long axis of the triangular cylinder located at $Y=0$, $-1,-3 \mathrm{~cm}$; the planes at $Y=-1 \mathrm{~cm}$ and $Y=-3 \mathrm{~cm}$ were $1 \mathrm{~cm}$ inside and outside the edge of the cylinder, respectively, which provided data for testing the threedimensional nature of an algorithm's heterogeneity correction. At $9 \mathrm{MeV}$, a BEV dose distribution was measured in a solid water phantom at a depth of $2.8 \mathrm{~cm}$ from the phantom surface. Using the $20-\mathrm{MeV}$ beam, planar $\mathrm{BEV}$ dose distributions were measured at depths of 3.1 and $6.1 \mathrm{~cm}$. A $15 \times 15-\mathrm{cm}$ applicator was used to ensure that the volume of interest (i.e., where the dose distribution is perturbed by the triangular cylinder) was not influenced by the beam's penumbra.

\section{J. Experiment 10: Internal heterogeneity (1-D), lung}

A major concern in chest wall irradiation is the penetration of electrons into the lung. Since the lung has a physical density of only about 0.3 , after penetrating the chest wall the remaining portion of the depth-dose curve can penetrate three times deeper in lung than that in unit density tissue. Hence, a slab phantom was designed to simulate the chest wall-lung geometry. 

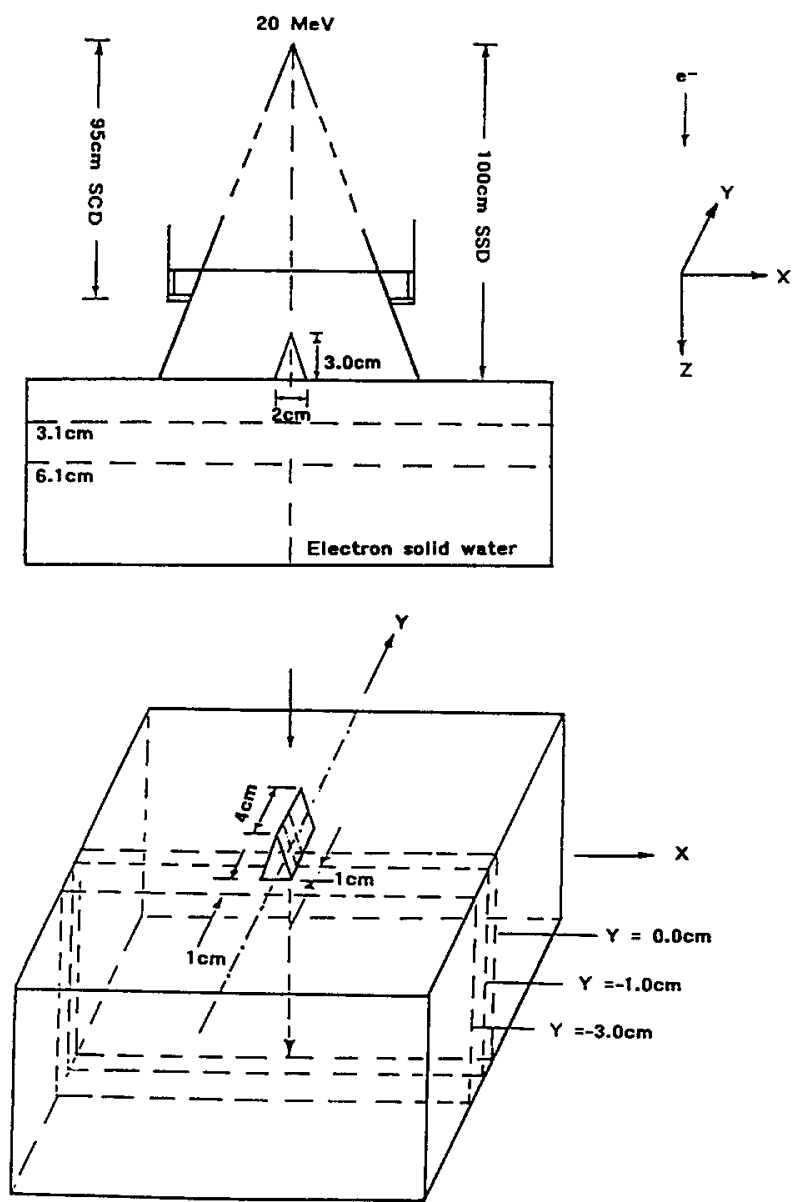

FIG. 7. Schematic of irradiation geometry of a nose treatment measurement for a $20-\mathrm{MeV}$ beam. The locations of the BEV dose distribution measurements at depths of 3.1 and $6.1 \mathrm{~cm}$ are indicated by the dashed lines shown on the side view. The locations of the transverse dose distribution measurements in the principal plane and in the planes 1 and $3 \mathrm{~cm}$ away from the principal plane are indicated by the dashed lines on the isometric view.

The measurement was performed with a $9 \mathrm{MeV}, 15$ $\times 15-\mathrm{cm}$ electron field incident on the phantom, consisting of a $3-\mathrm{cm}$ solid water slab followed by a series of $1-\mathrm{cm}$ thick lung-substitute slabs (see Fig. 8). The solid water slab and energy were selected to place the $90 \%$ depth dose at the solid water-lung substitute interface. The depth dose was measured every $1 \mathrm{~cm}$ along central axis.

\section{K. Experiment 11: Internal heterogeneity (2-D), lung- mediastinum interface}

Conventional electron pencil beam algorithms become less accurate for heterogeneity-unit density tissue interfaces that are long and parallel to the beam. ${ }^{6}$ This condition often exists at the lateral border between lung and the mediastinum. To simulate this patient geometry, a "semiinfinite" slab of lung substitute was abutted on central axis to "semi-infinite" slab of solid water; both slabs were covered by an additional $3-\mathrm{cm}$ thick slab of solid water. The irradiation geometry is diagrammed in Fig. 9. The internal mammary chain is usually irradiated with an electron beam energy in the range of $12-16 \mathrm{MeV}$; for this test a

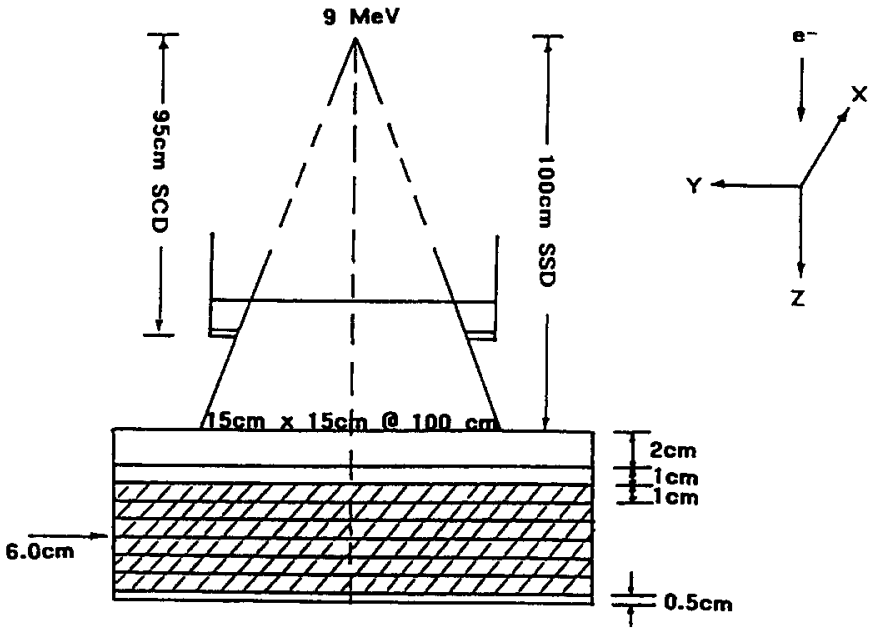

Electron Solid Water (RMI)

Lung Substitute Material (RMI)

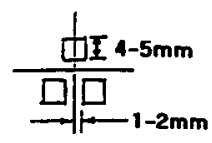

Beam's eye view

FIG. 8. Schematic of irradiation geometry of a series of 1-cm thick lung slabs within a solid water phantom for a $9-\mathrm{MeV}$ beam. The TLD placement with respect to the central axis of the beam is illustrated on the BEV.

20-MeV beam was used. BEV planar dose distributions were measured at depths of 5 and $7 \mathrm{~cm}$ from the surface, and a dose distribution was measured in a plane containing the central axis and perpendicular to the established interface in the solid water half of the phantom. Again, a 15 $\times 15-\mathrm{cm}$ field size was selected to ensure that the portion of the beam being studied was sufficiently inside the penumbra.

\section{Experiment 12: Internal heterogeneity (2-D), hard bone}

Hard bones just below the patient's skin surface can perturb the dose distribution resulting from electron beam irradiation. Irradiation through the mandible is the most frequently encountered case clinically, although other hard bones of the head, bones of the forearm or lower leg, and the spinal column are also of interest. The primary effects of hard bone on the dose distribution are twofold: (a) it pulls the isodose contours beneath it back toward the surface and (b) it generates volumes of increased/decreased dose just outside/inside its lateral edge. ${ }^{6,18,20}$ This patient geometry was simulated by a long bone slab $1-\mathrm{cm}$ thick by $3-\mathrm{cm}$ wide located $1 \mathrm{~cm}$ beneath the surface, since in this case, we were interested in the dose distribution beneath 

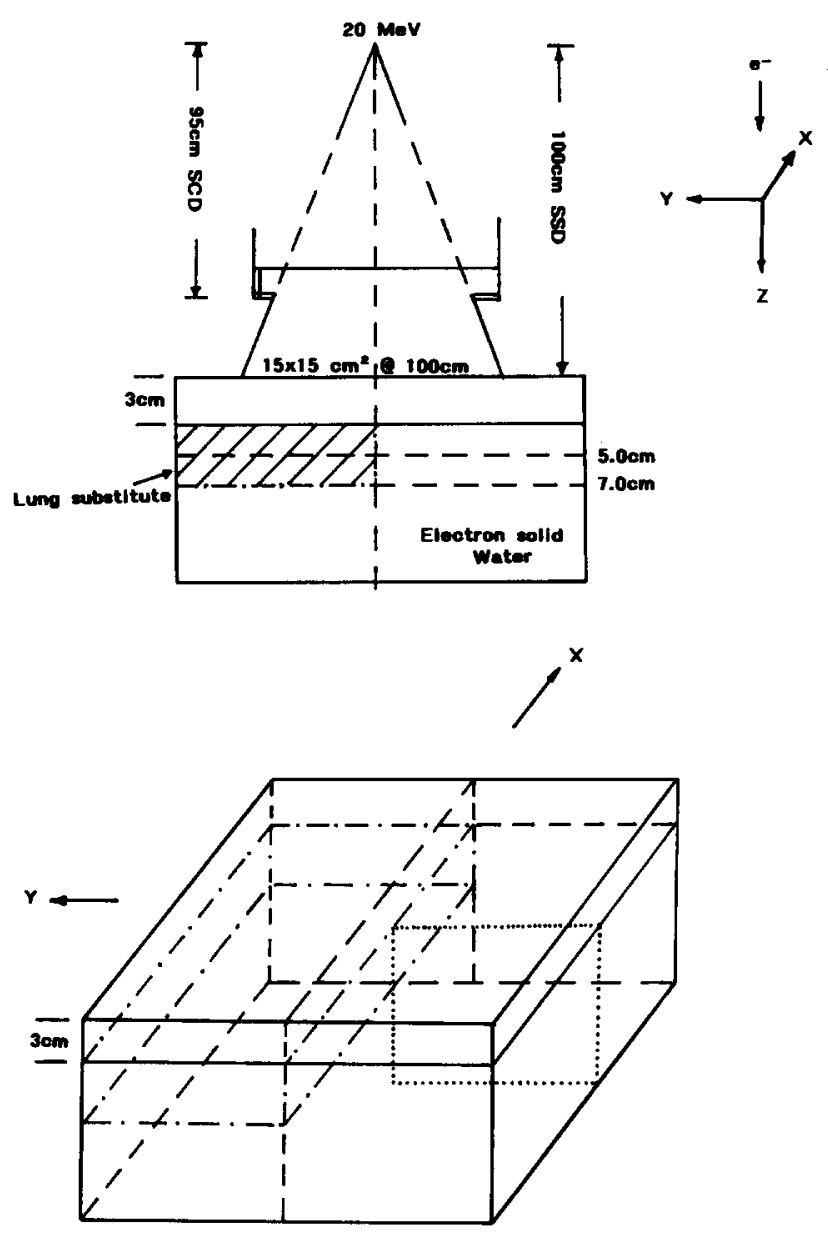

FIG. 9. Schematic of irradiation geometry of a quarter-infinite slab of lung within a solid water phantom for a $20-\mathrm{MeV}$ beam. The locations of the BEV dose distribution at depths of 5 and $7 \mathrm{~cm}$ are indicated by the dashed lines shown on the side view. The location of the transverse planar dose distribution measurement in the half principal plane in the electron solid water measurements is indicated by the dashed lines illustrated on the isometric view.

the bone. Dose distributions were measured beneath the bone with both energies. The $20-\mathrm{MeV}$ irradiation geometry is shown in Fig. 10.

\section{Experiment 13: Internal heterogeneity (2-D), air cavity}

Air cavities just below patient's skin surface can perturb the dose distribution resulting from electron beam irradiation. This type of perturbation is most often encountered in electron irradiations of the neck with energies sufficient to penetrate the trachea. The effect of a long cylindrical air cavity is twofold: (a) the isodose lines generated beneath the air cavity become more penetrating, and (b) the cavity consequently generates volumes of increased/ decreased dose just inside/outside its lateral edge. ${ }^{20}$ This patient geometry was simulated by an air cavity (styrofoam ) $1-\mathrm{cm}$ thick by $3-\mathrm{cm}$ wide located $1 \mathrm{~cm}$ beneath the surface. Dose distributions were measured below the air cavity for both energies, as depicted in Fig. 10.
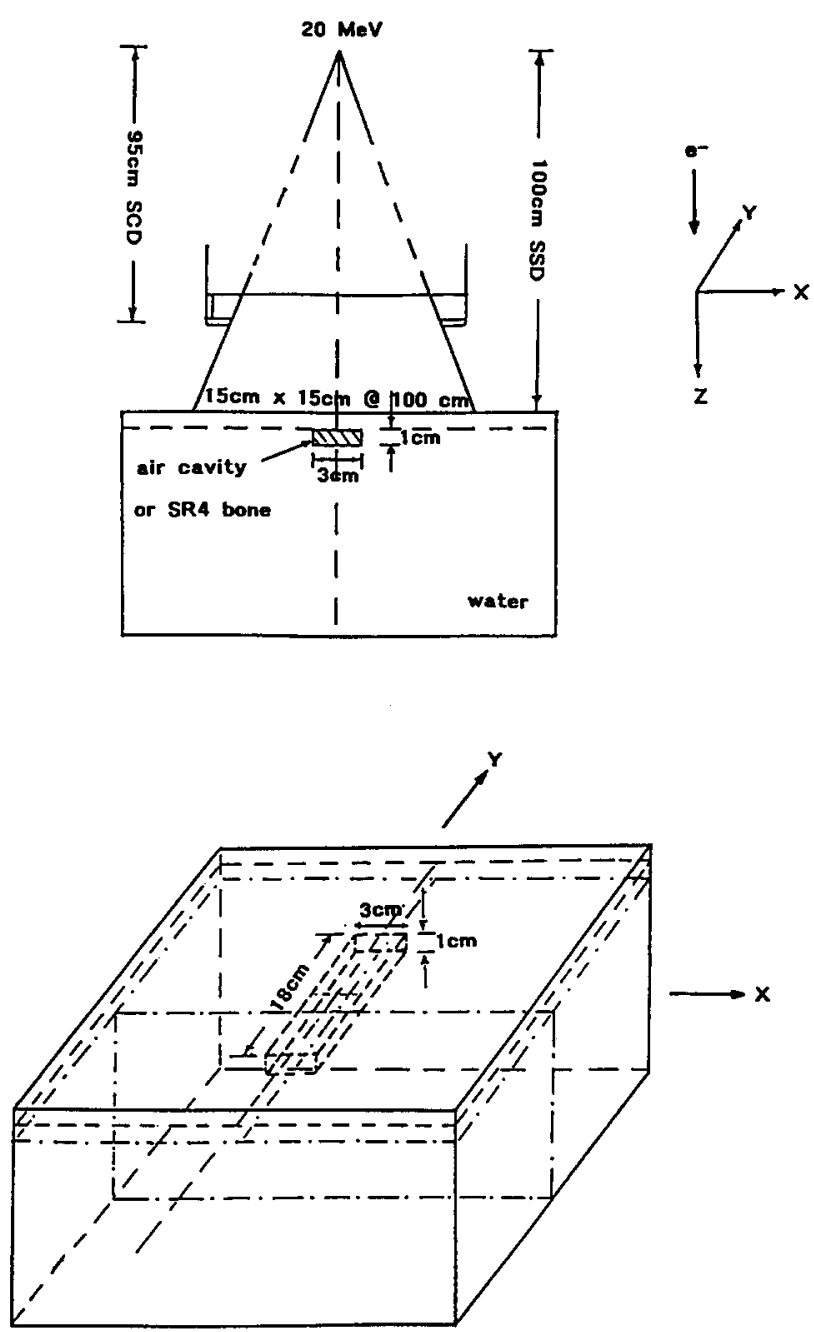

FIG. 10. Schematic of irradiation geometry of a long bone slab or a long air cavity $(1 \mathrm{~cm}$ thick by $3 \mathrm{~cm}$ wide) within a water phantom measurement for a $20-\mathrm{MeV}$ beam. The location of the principal plane that measured the transverse dose distribution beneath the bone slab or the air cavity is indicated by the dashed-dotted lines on the BEV.

\section{N. Experiment 14: Internal heterogeneity (3-D), ramus of mandible}

In treatment of head and neck cancers, the ramus of the mandible is often within the treatment field. Because this case does not meet the criterion of a "2-D heterogeneity" (i.e., not long in the dimension perpendicular to the plane of calculation as was the case in Experiment 12), one can expect a difference between a 2-D or 3-D heterogeneity-corrected algorithm in the accuracy of dose calculation to bone. The patient geometry for this test was simulated by an L-shaped bone slab $1-\mathrm{cm}$ thick by $3-\mathrm{cm}$ wide located $1 \mathrm{~cm}$ beneath the surface (Fig. 11). Axial dose distributions on the central axis, and $1 \mathrm{~cm}$ to each side of the edge of the " $L$ " were supplemented with the usual BEV planar dose distributions.

\section{RESULTS AND DISCUSSION}

The 14 experiments discussed in the text produced 78 planar dose distributions and 1 depth-dose curve. We have grouped these experiments into four categories: (1) basic 


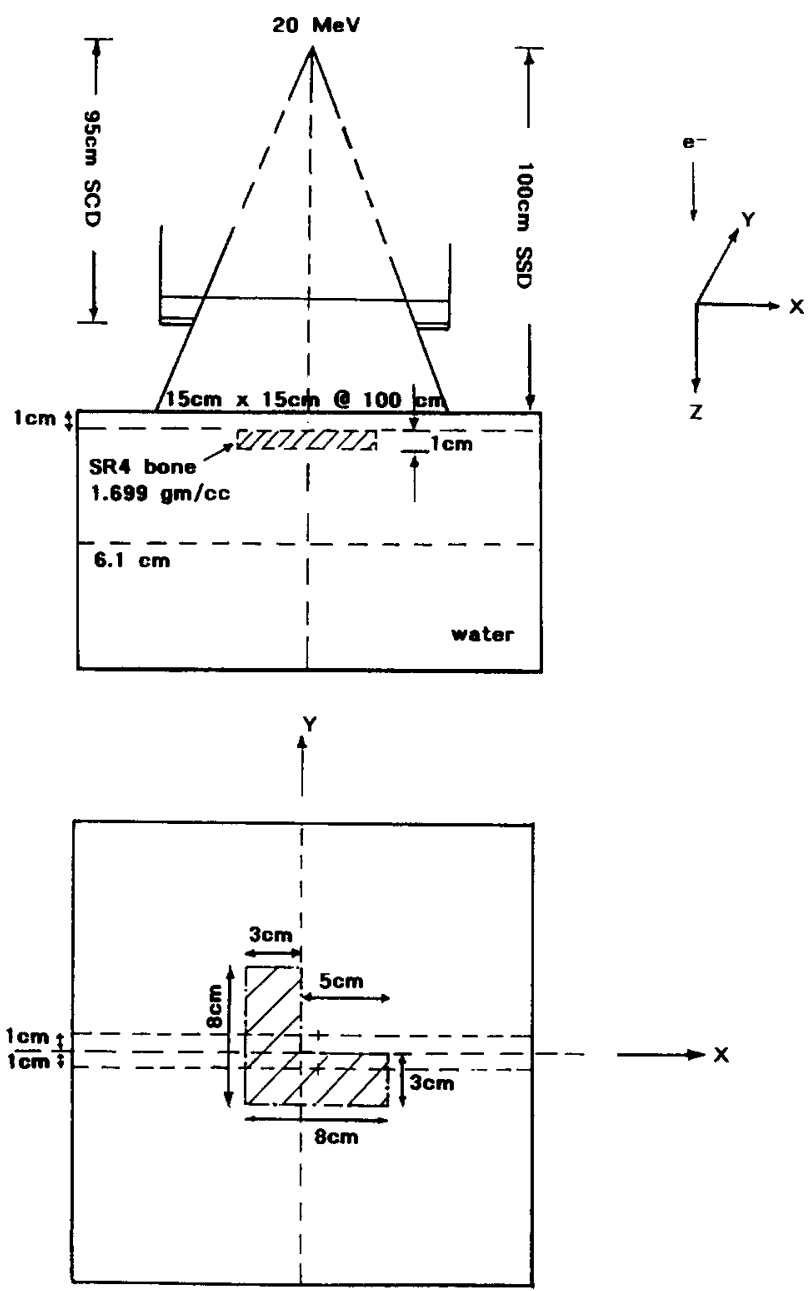

Fig. 11. Schematic of irradiation geometry of an L-shaped bone (ramus of mandible) within a water phantom for a $20-\mathrm{MeV}$ beam. The location of the BEV dose distribution measurement at depth of $6.1 \mathrm{~cm}$ is indicated by the dashed lines shown on the side view. The locations of the transverse dose distributions measured beneath the $\mathrm{L}$-shaped bone, $1 \mathrm{~cm}$ from either side of the principal transverse plane was indicated by the dashed lines on the BEV.

geometry, (2) field shaping, (3) oblique incidence and irregular surfaces, and (4) heterogeneous phantoms. The data generated from basic geometry were used by each of three institutions to ensure that their pencil-beam algorithms reproduced the central-axis depth doses exactly and that the input data and beam parameters were chosen correctly. Then, the rest of the measured data were used to evaluate the accuracy of the dose algorithm as a function of position in the beam due to the effects of field shaping, the beam incidence with respect to the patient surface, irregular patient surface, and internal inhomogeneous structures. Comparison of algorithm calculations with this remaining data set allowed members of ECWG to understand each of their algorithm's clinical applicability. Only selected results of the measured data set are shown in this paper; however, plots of all 79 dose distributions are available to accompany the magnetic tape distribution of data as well as a basic description of beam characteristics and a general guidance to the user with regard to extraction of algorithmic parameters from the data.
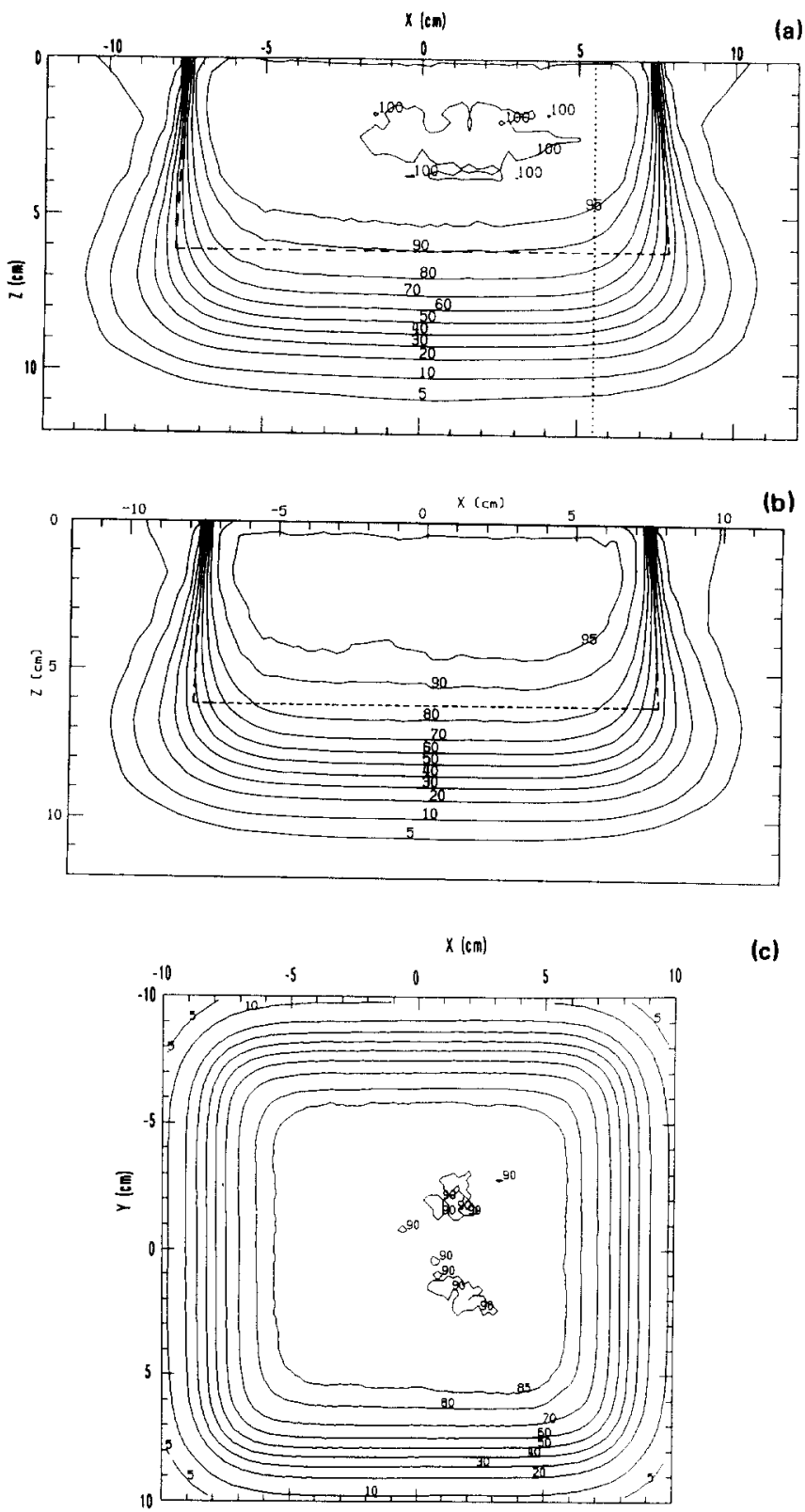

(c)

FIG. 12. The planar isodose contours from the $20-\mathrm{MeV}$ beam, $15 \times 15-\mathrm{cm}$ field at 100-cm SSD; (a) principal transverse plane, (b) transverse plane $2 \mathrm{~cm}$ away from beam edge, and (c) BEV at depth of $6.1 \mathrm{~cm}$.

\section{A. Basic geometry}

Two measured isodose contour plots in a plane parallel to central axis and the $X$ axis ( $Y=0, Y=$ beam edge-2 $\mathrm{cm}=5.5 \mathrm{~cm}$ ) for a $20 \mathrm{MeV}, 15 \times 15-\mathrm{cm}$ field in water at a 100-cm SSD are illustrated in Fig. 12(a) and (b), respectively (experimental setup illustrated in Fig. 1). The dotted line in Fig. 12(a) marks the location of the off-axis planar scan of Fig. 12(b) (where it is offset from the $X$ axis rather than the $Y$ axis). Assuming $X-Y$ symmetry, the depth dose along the dotted line agrees well with the depth dose at $X=0$ in Fig. 12(b). The dashed line in Fig. 12(a) shows the geometric treatment volume (defined by the projection of the light field edges and central-axis depth of $90 \%$ dose, $R_{90}$ ), for comparison to the actual area of the $90 \%$ isodose curve (used typically as the treatment pre- 


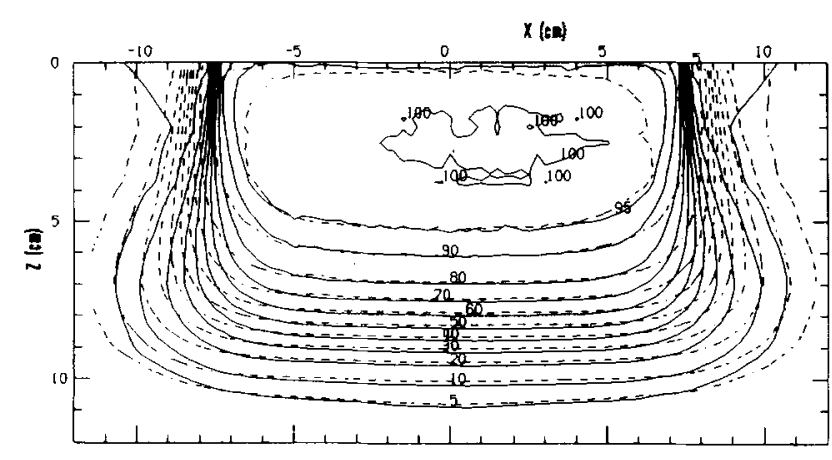

FIG. 13. Comparison of isodose contours at 100 and 110-cm SSD for a $15 \times 15-\mathrm{cm}, 20-\mathrm{MeV}$ beam. Each dose distributions is normalized to its own maximum dose along central axis.

scription isodose line). Particularly on Fig. 12(b), which shows a dose distribution obtained $2 \mathrm{~cm}$ from the field edge, one can note that the $90 \%$ isodose line is "pulled back" such that a region of decreased dose occurs in the corners of the geometric treatment volume. In both dose distributions, the spreading of the $5 \%$ isodose contour near the surface indicates that dose outside the penumbra is due to leakage of primary electrons through the collimator walls. Figure 12(c) shows the BEV planar dose distribution at $6.1-\mathrm{cm}$ depth for the $15 \times 15-\mathrm{cm}$ applicator at 20 $\mathrm{MeV}$ to illustrate the penumbra, flatness, and behavior near the corners of the applicator. These three dose distributions plus the BEV dose distributions near the surface (plot not shown) should provide the fundamental data to evaluate any algorithm's ability to predict the dose distribution in a water phantom at the standard SSD.

Comparison of planar dose distributions, each normalized to its central axis maximum, at 100- and 110-cm SSD for a $15 \times 15-\mathrm{cm}, 20-\mathrm{MeV}$ electron beam is depicted in Fig. 13. These data demonstrate little difference in relative dose along the central axis of the beam, but show both increased width and penumbra for the 110-cm SSD because of the increased air gap. Although not present for the geometry in Fig. 13, there can be a significant difference in the depth dose at an extended SSD if applicator scatter contributes significantly to the dose distribution at the nominal SSD $(100 \mathrm{~cm})$. This is seen on the comparison of the percent depth dose curves at 100 - and $110-\mathrm{cm}$ SSD for a $6 \times 6-\mathrm{cm}$ open applicator, 20-MeV electron beam [Fig. 14(a)], again both normalized to their individual maximum. Note, for example, how the decrease in scattered electrons for the $110-\mathrm{cm}$ geometry results in a decrease in the surface dose, a more uniform depth dose, and a deeper $R_{90}$. Consequently, the therapeutic portion of the beam (within $90 \%$ isodose contour) is much larger and deeper for the $110-\mathrm{cm}$ geometry as compared to that of the $100-\mathrm{cm}$ geometry and is shown on Fig. 14(b).

\section{B. Field shaping}

The BEV dose distribution at $20 \mathrm{MeV}$ for the "house block" inserted into the $15 \times 15-\mathrm{cm}$ applicator and measured at a depth of $6.1 \mathrm{~cm}\left(R_{90}\right.$ for the open applicator $)$ is shown in Fig. 15 (experimental setup illustrated in Fig. 2).
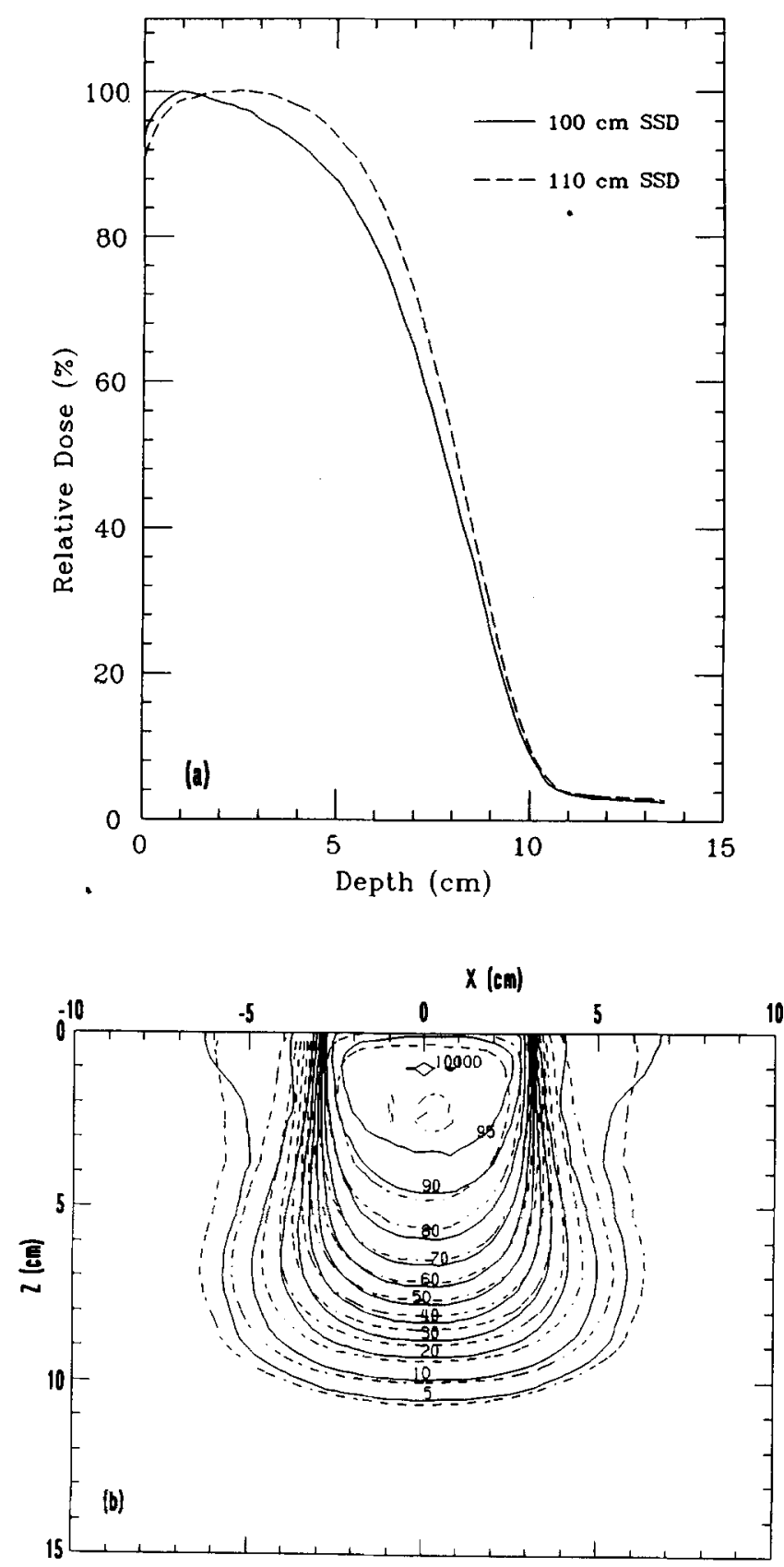

FIG. 14. Comparison of (a) central-axis depth-dose curves and (b) isodose contours at 100 - and $110-\mathrm{cm}$ SSD for a $6 \times 6-\mathrm{cm}$ applicator, $20-\mathrm{MeV}$ beam.

The data demonstrate a very small area within the $85 \%$ isodose contour as compared with the geometric area outlined by the light field. Side-scatter equilibrium does not exist in the "chimney" and "roof" areas of the "house block" field. The dose gradient varies from $85 \%$ to $20 \%$ within the geometric area. This result emphasizes the necessity of 3-D treatment planning to ensure that the desired target volume is enclosed by the therapeutic portion of the beam.

The central plane dose distribution for a $5-\mathrm{cm}$ diameter field, $9-\mathrm{MeV}$ beam measured at a $97-\mathrm{cm}$ SSD, is shown in Fig. 16. A 7-mm-thick lead, 1-cm diameter lens block was placed in the center of the circular insert (experimental 


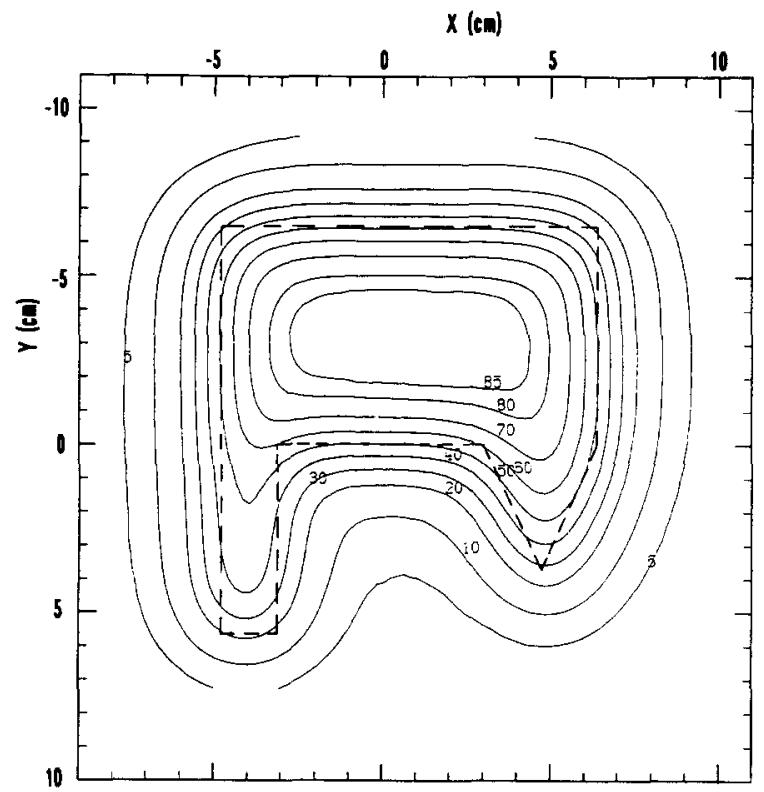

FIG. 15. The BEV dose distribution at depth of $6.1 \mathrm{~cm}$ for the 20-MeV beam, $15 \times 15-\mathrm{cm}$ applicator with the "house block" insert.

setup illustrated in Fig. 4). The dose distribution demonstrates that the multiple Coulomb scattering of electrons, as they enter the phantom, accounts for the "fill-in" of dose behind the lens block at depth.

\section{Oblique incidence and irregular patient surfaces}

Figure 17 documents a measured dose distribution for a $30^{\circ}$ oblique incidence, $20-\mathrm{MeV}$ electron beam set at SSD of $104.3 \mathrm{~cm}$ (experimental setup illustrated in Fig. 5). This plot shows the change in the penumbra and depth dose due to angulation of electron beam. Penumbral width is less in

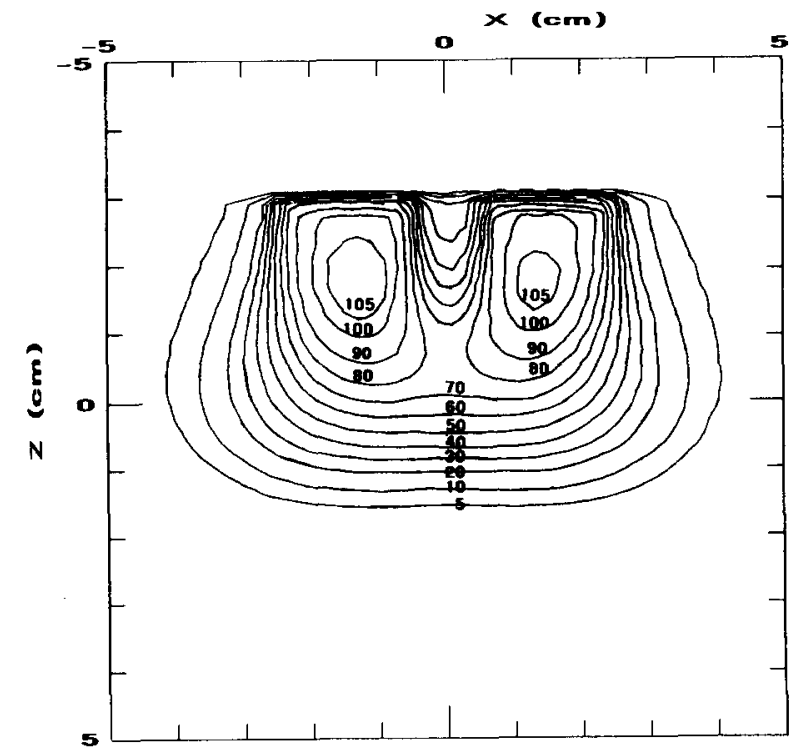

FIG. 16. Dose distribution for a 5-cm diameter circular field with a lens block (lead) placed at the center of the field; $9-\mathrm{MeV}$ beam at $97-\mathrm{cm} \mathrm{SSD}$; 95-cm source to lens block distance.

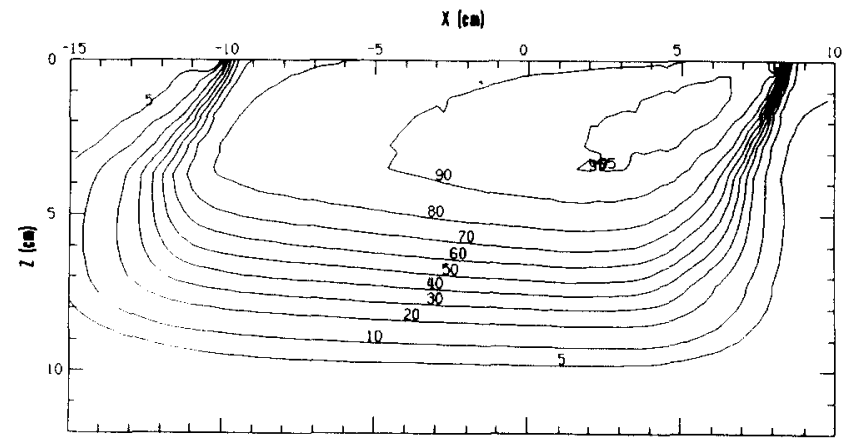

FIG. 17. Isodose contours for the $15 \times 15-\mathrm{cm}, 20-\mathrm{MeV}$ beam for a $30^{\circ}$ oblique incidence.

the region of the surface with the lesser air gap. In general, the depth dose is affected in three ways: (1) dose increases near the surface, (2) the therapeutic depth $\left(R_{90}\right)$ decreases, and (3) the practical range $\left(R_{p}\right)$ increases.

The data displayed in Fig. 18 illustrate the effect of the "nose" phantom (experimental setup illustrated in Fig. 6). The measured dose distribution in a plane parallel to the central axis and perpendicular to the long axis of the triangular "nose" located at $Y=0.0$ is shown in Fig. 18(a). Areas of increased dose (hot spots) are located inferior
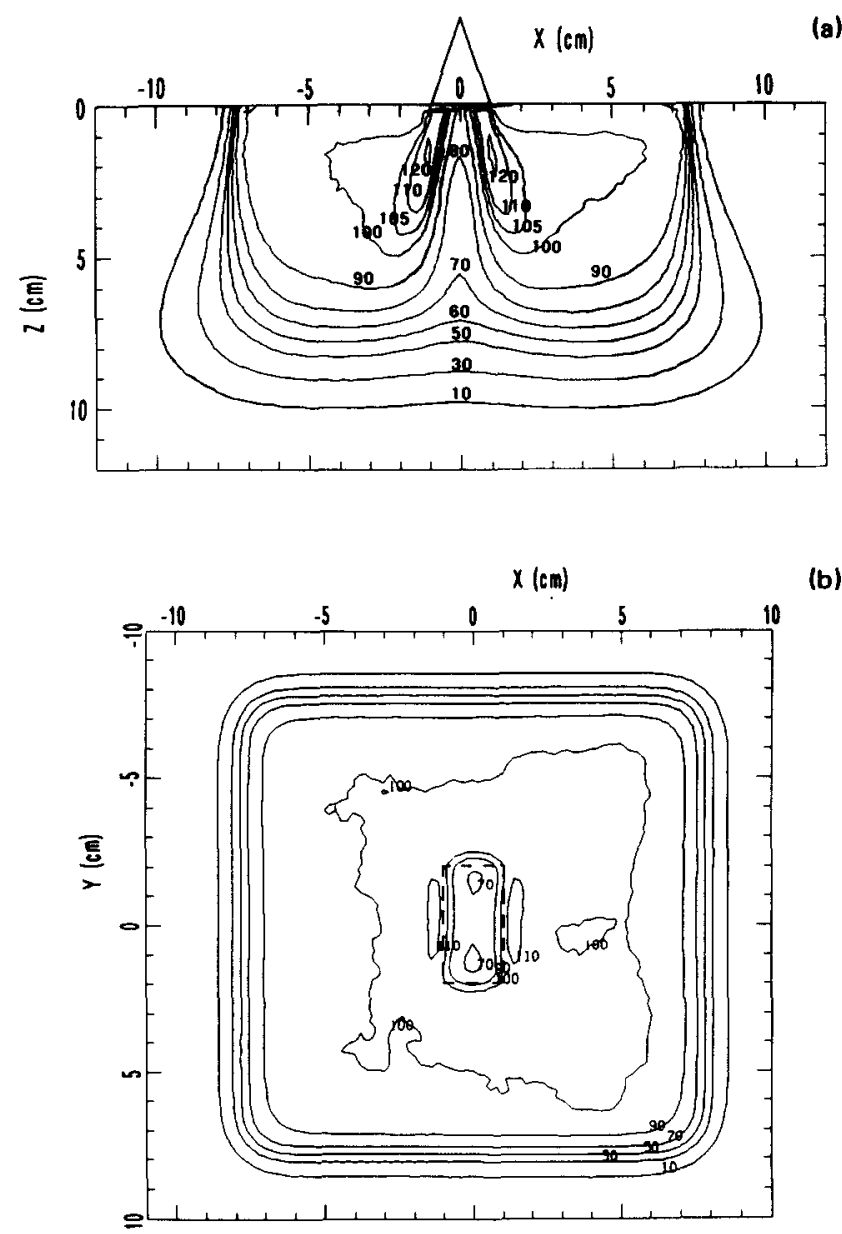

(b)

FIG. 18. The 20-MeV beam's dose distributions (a) for the principal transverse plane located perpendicular to the long axis of the triangular cylinder (nose) and (b) for the BEV plane located at a depth of $3.1 \mathrm{~cm}$. 


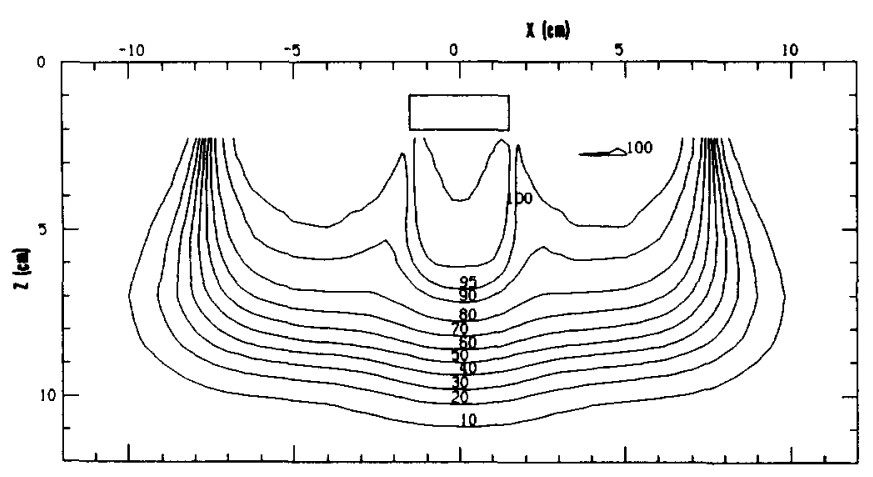

FIG. 19. A transverse plane dose distribution beneath a 1-cm thick air cavity (styrofoam) located at depth of $1 \mathrm{~cm}$ from the surface of water phantom (20-MeV beam, $15 \times 15-\mathrm{cm}$ field, $100-\mathrm{cm} \mathrm{SSD}$ ).

and lateral to the nose and the corresponding areas of decreased dose (cold spots) are beneath the nose. The measured BEV plane dose distribution for $20-\mathrm{MeV}$ electrons at depth of $3.1 \mathrm{~cm}$ is shown in Fig. 18(b).

\section{Heterogeneous phantom}

For a $20-\mathrm{MeV}$ beam, isodose curves measured in water behind a $1-\mathrm{cm}$ thick air cavity (styrofoam) with the proximal surface situated at a depth of $1 \mathrm{~cm}$ from the surface of water (experimental setup illustrated in Fig. 10) are plotted in Fig. 19. Behind the lateral edges of the air cavity, the dose is increased inside the lateral edges and decreased outside because less electrons are scattered out by the air than are scattered in by the water.

The measurements beneath the 3-D (L-shaped) bone phantom (experimental setup illustrated in Fig. 11) provide valuable data to evaluate an algorithm's ability to predict the dose beneath a 3-D inhomogeneity. The dose distribution measured in a plane $1-\mathrm{cm}$ away from the central axis of the beam and cutting through the shorter width of the bone is illustrated in Fig. 20(a). The dose enhancement due to adjacent bone $1 \mathrm{~cm}$ outside the plane of calculation is very pronounced in this distribution. Figure $20(\mathrm{~b})$ is the BEV dose distribution at a depth of $6.1 \mathrm{~cm}$. These data illustrate in the two lateral dimensions the areas of increased/decreased dose just outside/inside the edges of the bone.

\section{SUMMARY}

This article presents a set of measured electron beam dose distributions designed for extensive electron beam dose algorithm verification. In contrast to some earlier sets of measurements, the experiments reported here were designed to simulate a number of common electron beam dosimetry issues that arise during routine electron beam treatment and treatment planning. The effects of energy, field size, field shaping, SSD, irregular patient surfaces, and inhomogeneities (1-D, 2-D, and 3-D) have all been studied.

This data set was designed, measured, and analyzed by the three members of the ECWG. As part of the work of


FIG. 20. For the $20-\mathrm{MeV}$ beam, $15 \times 15-\mathrm{cm}$ field, $100-\mathrm{cm} \mathrm{SSD}$, the dose distributions beneath a $1-\mathrm{cm}$ thick L-shaped bone located (a) in a transverse plane $1 \mathrm{~cm}$ away from the central axis of the beam, cutting through the shorter width of the bone, and (b) in a BEV plane at a depth of 6.1 $\mathrm{cm}$.

the ECWG, the measured data set has been transferred electronically to all three institutions, and then used by each in their electron dose algorithm studies. It has provided sufficient measured data for comparison with calculations from a wide variety of electron beam algorithms for the purpose of evaluating their clinical applicability. In order to accomplish this cooperative work, the measurements and computerized data set were carefully set up, analyzed, and documented. As the transfer mechanisms have already been tested by the three groups, the ECWG decided to make this data set available to interested institutions, both on paper and on magnetic tape. The distribution of these data should be useful to a wide audience of professionals, who are developing an electron beam algorithm or implementing them into treatment planning systems.

\section{ACKNOWLEDGMENT}

This investigation was supported in part by NCI Contract Nos. N01-CM-67913, 4, and 5.

\footnotetext{
${ }^{1}$ A. Brahme, "Standard geometry for comparison of dosimetry and dose planning programs for electron beams," Acta Radiol. Suppl. 364, 101102 (1983).
} 
${ }^{2}$ K. R. Shortt, C. K. Ross, A. F. Bielajew, and D. W. Rogers, "Electron beam dose distributions near standard inhomogeneities," Phys. Med. Biol. 31, 235-249 (1986).

${ }^{3}$ D. R. White, R. J. Martin, and R. Darlison, "Epoxy resin based tissues," Br. J. Radio. 5, 814-821 (1977).

${ }^{4} \mathrm{C}$. Constantinou, "Tissue substitutes for particulate radiations and their use in radiation dosimetry and radiotherapy," Ph.D. thesis, University of London (1978).

${ }^{5}$ K. R. Hogstrom, M. D. Mills, and P. R. Almond, "Electron beam dose calculations," Phys. Med. Biol. 26, 445-459 (1981).

${ }^{6}$ K. R. Hogstrom, M. D. Mills, J. A. Meyer, J. R. Palta, D. E. Mellenberg, R. T. Meoz, and R. S. Field, "Dosimetric evaluation of a pencilbeam algorithm for electrons employing a two-dimensional heterogeneity correction," Int. J. Radiat. Oncol. Biol. Phys. 10, 561-569 (1984).

${ }^{7}$ R. A. Keys and J. A. Purdy, "Radiation leakage from linac electron applicator assembly," Int. J. Radiat. Oncol. Biol. Phys. 10, 713-721 (1984).

${ }^{8}$ E. C. Pennington, S. K. Jani, and B. C. Wen, "Leakage from electron applicators on a medical accelerator," Med. Phys. 15, 763-765 (1988). ${ }^{9}$ M. H. Maor, R. S. Fields, K. R. Hogstrom, and J. Van Eys, "Improving the therapeutic ratio of craniospinal irradiation in medulloblastoma," Int. J. Radiat. Oncol. Biol. Phys. 11, 687-707 (1985).

${ }^{10}$ L. Dewit, J. Van Dam, A. Rijnders, G. Van De Velde, K. K. Ang, and E. Van Der Schueren, "A modified radiotherapy technique in the treatment of medulloblastoma," Int. J. Radiat. Oncol. Biol. Phys. 10, 231241 (1984)

${ }^{11}$ C. Constantinou, F. H. Attix, and B. R. Paliwal, "A solid water phantom material for radiotherapy $\mathrm{x}$-ray and $\gamma$-ray beam calibrations," Med. Phys. 9, 436-441 (1982).

${ }^{12}$ International Commission on Radiation Units and Measurements, Radiation Dosimetry: Electrons with Initial Energies Between 1 and 50
MeV, ICRU Report 21, 1972 (International Commission on Radiation Units and Measurement, Bethesda, MD.)

${ }^{13}$ R. K. Ten Haken, B. A. Fraass, and R. J. Jost, "Practical methods of electron depth-dose measurements compared to use of the NACP design chamber in water," Med. Phys. 14, 1060-1066 (1987).

${ }^{14} \mathrm{~J}$. Milan and R. E. Bentley, "The storage and manipulation of radiation dose data in a small digital computer," Br. J. Radiol. 47, 115-121 (1974).

${ }^{15}$ A. S. Shiu, V. A. Otte, and K. R. Hogstrom, "Measurement of dose distributions using film in therapeutic electron beams," Med. Phys. 16, 911-915 (1989).

${ }^{16}$ A. S. Shiu and R. K. Hogstrom, "Dose in bone and tissue near bonetissue interface from electron beam," Int. J. Radiat. Oncol. Biol. Phys. 21, 695-702 (1991).

${ }^{17}$ S. M. Kirsner, K. R. Hogstrom, R. G. Kurup, and M. R. Moyers, "Dosimetric evaluation in heterogeneous tissue of anterior electron beam irradiation for treating retinoblastoma," Med. Phys. 14, 772-779 (1987).

${ }^{18}$ K. R. Hogstrom and P. R. Almond, "Comparison of experimental and calculated dose distributions: A review of electron beam dose planning at the M. D. Anderson Hospital," Acta Radiol. Supp. 364, 89-99 (1983).

${ }^{19}$ K. E. Ekstran and R. L. Dixon, "The problem of obliquely incident beams in electron beam treatment planning," Med. Phys. 9, 276-278 (1982).

${ }^{20}$ K. R. Hogstrom, "Dosimetry of electron heterogeneities," in Medical Physics Monograph No. 9: Advances in Radiation Therapy Treatment Planning, edited by A. Wright and A. Boyer (American Institute of Physics, New York, 1983), p. 223.

${ }^{21}$ N. duV. Tapley, "Skin and Lips," in Clinical Applications of the Electron Beam, edited by N. duV. Tapley (Wiley, New York, 1976). 